ИЗВЕСТИЯ АКАДЕМИИ НАУК ЭСТОНСКОП ССР. ТОМ 32 ФИЗИКА * МАТЕМАТИКА. 1983, № 3

\title{
О ВОЗМОЖНОСТИ ПОСТРОЕНИЯ КОНТРМОДЕЛИ ДЛЯ НЕДЕТЕРМИНИРОВАННОЙ ФОРМУЛЫ *
}

\author{
(Представил Н. Алумяэ)
}

Рассмотрим, в каком случае для формулы исчисления предикатов высших порядков (может быть, бесконечной) можно построить модель, истинностное значение которой при некоторой интерпретации этой формулы отличается от единицы. Исходя из результатов, приведенных в [ $\left.{ }^{1}\right]$, мы можем ограничиться моделями в виде семантической структуры.

B $\left[{ }^{2}\right]$ показано, что для недетерминированной в смысле $\left[{ }^{3}\right]$ формулы можно при каждом множестве $\mu$ термов найти $\mu$-нормальную контрмодель. 'Но если $\mu$ не множество, а класс всех термов, то указанная в $\left[{ }^{2}\right]$ процедура дает модель в виде собственного класса. Чтобы модель была множеством, достаточно, чтобы аспекты и индивиды составляли множество, так как в этом случае и совокупность предикатов произвольного заданного типа будет множеством.

В принципе возможны три случая:

1) для каждой недетерминированной формулы можно построить контрмодель, где аспекты и индивиды всех типов образуют множество;

2) для каждой недетерминированной формулы можно построить контрмодель, где аспекты образуют множество, но для некоंторых формул индивиды в этом случае обязательно образуют собственный класс;

3) для некоторой недетерминированной формулы в каждой контрмодели аспекты образуют собственный класс.

Легко можно доказать, что случай 1) не вытекает из аксиоматики Цермело-Френкеля. Например, для формулы

$$
\begin{aligned}
& \urcorner\{\forall P\{\forall\langle x, y\rangle(P x \wedge R y x \rightarrow P y) \rightarrow \forall x P x \vee \exists x \quad \forall y[x=y \leftrightarrow \\
& \leftrightarrow \forall z(R z y \leftrightarrow P z)]\} \wedge \forall Q[\forall\langle x, y, z\rangle(Q x y \wedge Q x z \rightarrow y=z) \wedge \\
& \wedge \exists z \forall x(\exists y Q x y \rightarrow R x z) \rightarrow \exists z \forall y(\exists x Q x y \rightarrow R y z)] \wedge \\
& \wedge \forall \Re\{\forall\langle P, u, v\rangle(\Re P u \wedge \Re P v \rightarrow u=v) \wedge \exists z \forall P[\exists u \Re P u \rightarrow \\
& \rightarrow \forall v(P v-R v z)] \rightarrow \exists z \forall u(\exists P \Re P u \rightarrow R u z)\} \wedge \\
& \wedge \exists x\{\exists y R y x \wedge \forall y[R y x \rightarrow \exists z(R y z \wedge R z x)]\}\}
\end{aligned}
$$

контрмодель может быть множеством только в том случае, если существует сильно недостижимый кардинал $\boldsymbol{\aleph}>\boldsymbol{\aleph}_{0}$.

Будем сравнивать случаи 2) и 3), полагая, что аксиоматика теории множеств содержит аксиоматику Цермело-Френкеля.

Зафиксируем $\mathfrak{A}-$ некоторую недетерминированную формулу, для которой нормальной контрмодели, являющейся множеством, не су-

\footnotetext{
* Предполагается, что читатель знаком с содержанием статей [1-4].
} 
ществует. В дальнейшем все определяемые понятия й получаемые результаты относятся именно к данной формуле भ. При этом будем по-разному рассматривать случаи, когда $\mathscr{U}$ имеет некоторую бесконечную мощность $\mathfrak{乛}$ и когда $\mathfrak{A}$ конечна (этот случай обычно указываем в скобках). Будем говорить только о типах формулы $\mathfrak{A}$.

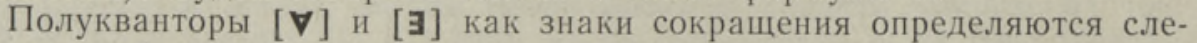
дующим образом.

Если $\left\langle x^{\prime}{ }_{t}: \iota \in J\right\rangle-$ некоторое семейство индивидов, то [ $\left.\boldsymbol{\nabla}\right]$

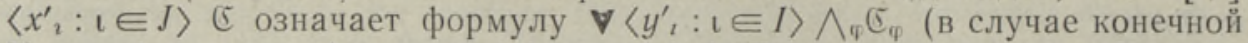
$\mathfrak{A}$ - формулу $\left.\bigwedge_{1 \leqslant n \in N} \forall\left\langle y_{2}^{\prime}: \iota \in I_{n}\right\rangle \bigwedge_{\varphi} \mathfrak{E}_{\varphi}\right)$, где $\left\langle y^{\prime}{ }_{2}: \iota \in I\right\rangle$ есть семейство, содержащее для каждого элементарного типа переменных $x_{2}, \imath \in J$, точно $\aleph$ переменных $\left(\left\langle y_{2}^{\prime}: \iota \in I_{n}\right\rangle\right.$ содержит $n$ переменных для каждого из указанных типов), ф пробегает все такие функции из $J$ в $I\left(\right.$ в $\left.I_{n}\right)$, при которых $x_{i}$ и $y_{\varphi(2)}$ принадлежат одинаковому типу, а $\mathfrak{F}_{\varphi}$ получается из 5 заменой переменных $x_{2}$ на $y_{\varphi(2)}$.

Запись $[\mathbf{a}]\left\langle x_{\imath}: \iota \in J\right\rangle \mathcal{E}$ означает формулу $\mathbf{3}\left\langle y_{i}^{\prime}: \iota \in I\right\rangle \bigvee_{\varphi} \mathfrak{夭}_{\varphi} \quad$ (в случае конечной $\mathscr{N}$ - формулу $\bigvee_{1 \leqslant n \in N} \exists\left\langle y_{2}^{\prime}: \imath \in I_{n}\right\rangle \bigvee_{\varphi}\left(_{\varphi}\right)$, где все символы имеют вышеуказанное значение.

Пусть $P$ - предикатная переменная или константа некоторого типа $\left\langle a_{\imath}^{\prime \prime}: \iota \in I, b_{\imath}^{\prime \prime}: \iota \in J\right\rangle$, где $a_{i}^{\prime \prime}-$ неэлементарные, а $b_{i}^{\prime \prime}-$ элементарные типы. Определим значение записи $[P]^{*}$, применяя индукцию по типу.

Если при $\iota \in I$ имеет место $a^{\prime \prime}{ }_{2}=\left\langle c^{\prime \prime}{ }_{x}: x \in K_{\imath}, d^{\prime \prime}{ }_{x}: x \in L_{1}\right\rangle$, где $c^{\prime \prime}{ }_{x}$ - неэлементарные, а $d^{\prime \prime}{ }_{x}$ - элементарные типы, то $[P]^{*}$ означает формулу

$$
\begin{aligned}
& {[\forall]\left\langle x^{\prime}: \iota \in J\right\rangle \forall\left\langle P^{\prime}{ }_{i}: \iota \in I, P_{2}^{\prime \prime}: \iota \in I\right\rangle\left\{\bigwedge _ { \imath \in I } \left[\left[P^{\prime}\right]^{*} \wedge\left[P_{i}^{\prime \prime}\right]^{*} \wedge\right.\right.} \\
& \bigwedge[\forall]\left\langle y_{x}^{\prime}: x \in L_{2}\right\rangle \forall\left\langle T_{x}: x \in K_{2}\right\rangle\left[\bigwedge_{x \in K_{1}}\left[T_{x}\right]^{*} \rightarrow\right. \\
& \left.\left.\rightarrow\left(P^{\prime}\left\langle T_{x}: x \in K_{\imath}, y_{x}^{\prime}: x \in L_{2}\right\rangle \leftrightarrow P^{\prime \prime}\left\langle T_{x}: x \in K_{\imath}, y_{\kappa}^{\prime}: x \in L_{2}\right\rangle\right)\right]\right] \rightarrow \\
& \left.\rightarrow\left(P\left\langle P^{\prime}{ }_{2}: \imath \in I, x^{\prime}{ }_{\imath}: \imath \in J\right\rangle \leftrightarrow P\left\langle P^{\prime \prime}: \imath \in I, x_{2}^{\prime}: \imath \in J\right\rangle\right)\right\} .
\end{aligned}
$$

Будем называть формулу умеренной, если в ней имеется не более чем $\aleph$ (конечное число) индивидных констант, каждый квантор существования и каждый квантор общности связывают не более чем $\boldsymbol{\aleph}$ (конечное число) индивидов, а применение таких кванторов по некоторому предикату $T$ всегда связано с релятивизацией их относительно $[T]^{*}$. Терм будем называть умеренным, если он элементарного типа или если его матрица - умеренная формула.

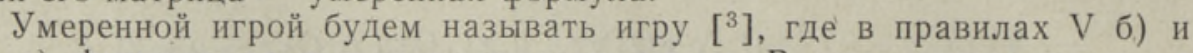
VI a) фигурируют только умеренные термы. В этом случае вместе с предикатной константой $Q$, полученной устранением квантора общности или квантора существования из матрицы умеренного терма, всегда возникает и выражение вида $\left([Q]^{*}\right)_{\beta}$. Но тогда применение правила VII c такой константой $Q$ можно заменить применением других правил на $\left([Q]^{*}\right)_{\beta}$. Из этого вытекает, что в хөде умеренной игры возникает ситуация, когда при каждом аспект-символе имеется лишь $\mathbf{\aleph}$ (конечное число) индивидных констант. Такая ситуация при использовании процедуры из $\left[{ }^{2}\right]$ индуцирует модель, являющуюся собственным классом, нормальную относительно класса умеренных термов - в дальнейшем такие модели будем называть умеренно нормальными - и содержа-

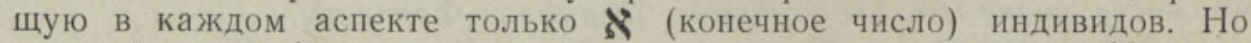
каждый терм $a_{1}^{\prime}$ можно преобразовать в умеренный терм $a_{2}^{\prime}$ путем замены кванторов общности и существования, связывающих индивиды, на соответствующие полукванторы и релятивизации относительно [T]* аналогичных кванторов, связывающих предикаты $T$. Такой терм 
$a_{2}^{\prime}$ в вышеуказанной модели эквивалентен $a_{1}^{\prime}$. Поэтому модель оказывается нормальной.

Теперь, исходя из вышеупомянутой формулы $\mathfrak{A}$, определим формулу $\mathfrak{B}$. Пусть $v$ - любое подмножество множества элементарных типов формулы $\mathfrak{U}$. Тогда $\mathfrak{B}_{v}$ определяется как формула

$$
\begin{gathered}
\forall\left\langle x_{\imath}^{\prime}: \iota \in J\right\rangle \exists\left\langle y_{\imath}^{\prime}: \iota \in I\right\rangle \bigvee_{\varphi}\left[\bigwedge _ { K } \forall P \left(P\left\langle x_{x}^{\prime}: x \in K\right\rangle \leftrightarrow\right.\right. \\
\left.\left.\leftrightarrow P\left\langle y_{\varphi(x)}^{\prime}: x \in K\right\rangle\right)\right]
\end{gathered}
$$

(в случае конечной $\mathfrak{U}$ - как формула

$$
\left.\forall\left\langle x^{\prime}{ }_{\imath}: \iota \in J\right\rangle \bigvee_{1 \leqslant n \in N} \exists\left\langle y^{\prime}{ }_{\imath}: \iota \in I_{n}\right\rangle \bigvee_{\varphi}\left[\bigwedge_{\imath \in J} \forall P\left(P x^{\prime}{ }_{\imath} \leftrightarrow P y_{\varphi \varphi(2)}\right)\right]\right),
$$

где $\left\langle x_{\imath}^{\prime}: \iota \in J\right\rangle$ содержит $\boldsymbol{\aleph}^{+}$(счетное множество) переменных каждого типа из $v$, а $\left\langle y^{\prime}{ }_{2}: \iota \in I\right\rangle-\boldsymbol{\aleph}$ переменных каждого из этих типов $\left(\left\langle y^{\prime}{ }_{2}: \iota \in I_{n}\right\rangle\right.$ содержит $n$ переменных каждого типа из $\left.v\right)$, ф пробегает все отображения, сохраняющие типы, из $J$ в $I$ (в $\left.I_{n}\right), K$ пробегает все подмножества множества $J$, а $P$ является предикатной переменной подходящего типа.

Пусть $\mathfrak{B}$ имеет вид $\bigwedge \bigwedge^{B}{ }_{v}$, где $v$ пробегает все подмножества множества элементарных типов данной сигнатуры.

Т еорем а 1. Формулӓ $\mathfrak{B} \rightarrow \mathfrak{H}-$ недетерминированная.

Доказательство следует из того, что в описанной выше контрмодели формулы $\mathfrak{A}$, которая является собственным классом и в каждом аспіекте которой существует только $\boldsymbol{\aleph}$ (конечное число) индивидов, формула $\mathfrak{B}$ истинна во всех аспектах.

Т е оре м а 2. Для $\mathfrak{A}$ не существует умеренно нормальной контрмодели, являющейся множеством.

Доказ ательст во. Сначала покажем, как из такой модели $M$ построить умеренно нормальную контрмодель для $\mathfrak{U}$, аспекты которой образуют множество, а в каждом аспекте существует не более чем $\aleph$ (конечное число) индивидов.

В новой модели каждому индивиду $а$ модели $M$ соответствуют два индивида $a^{+}$и $a^{-}$. Аспектами новой модели будут пары $(\beta, v)$, где $\beta-$ аспект модели $M$, а $v-$ множество, содержащее не более $\mathfrak{\aleph}$ (конечное число) индивидов, имеющихся в аспекте $\beta$, причем каждый элемент в $v$ снабжен некоторым образом знаком + или - . В аспекте $(\beta, v)$ будут существовать индивиды из $v$, а из объектов более высокого типа - те объекты $P$ из модели $M$, для которых в $\beta$ истинно $[P]^{*}$. Порядок аспектов определяется так, что $\left(\beta_{1}, v_{1}\right) \leqslant\left(\beta_{2}, v_{2}\right)$, если $\beta_{1} \leqslant \beta_{2}$ и $v_{1} \subseteq v_{2}$. Направлениями в $(\beta, v)$ будут, во-первых, направления в $\beta$, а во-вторых - множества $v_{0}$, содержащие не более $\boldsymbol{\aleph}$ (конечное число) индивидов, существующих в аспекте $\beta$ модели $M$ и не принадлежащих $v$. Непосредственными конкретизациями аспекта $(\beta, v)$ в первом случае будут пары $\left(\beta^{\prime}, v\right)$, где $\beta^{\prime}-$ непосредственная конкретизация аспекта $\beta$ в данном направлении, а во втором случае - пары $\left(\beta, v^{\prime}\right)$, где в роли $v^{\prime}$ выступают расширения множества $v$, в которых каждый элемент множества $v_{0}$ снабжен знаком + или -

Истинностные значения атомарных предложений в $(\beta, v)$ совпадают по определению с истинностными значениями их оригиналов в $\beta$.

Эта модель оказывается правильной. Индукцией по построению формулы доказывается, что каждая умеренная формула в аспекте $(\beta, v)$ имеет то же истинностное значение, что и в аспекте $\beta$ модели $M$, если значениями констант в $\beta$ являются оригиналы значений этих констант в $(\beta, v)$. Из этого вытекает, что новая модель умеренно нормальна и является контрмоделью для $\mathfrak{A}$. 
Но так как в каждом аспекте построенной модели существует не более чем $\boldsymbol{\aleph}$ (конечное число) индивидов и для каждого объекта $P$ более высокого типа $[P]^{*}$ истинна во всех аспектах, где $P$ существует, то семантика формул не меняется, если их преобразовать в умеренные путем замены кванторов по индивидам на полукванторы и релятивизации кванторов по переменным $P$ более высокого типа относительно $[P]^{*}$. Следовательно, новая модель и нормальна. Полученное противоречие с нашими предположениями доказывает теорему.

Т еорем а 3. Для формулы $\mathfrak{B} \rightarrow \mathfrak{A}$ не сущцествует нормальной контрмодели, аспекты которой составляли бы множество.

Доказ ательство. Предположим, что такая модель $M$ существует. Тогда для некоторого $\alpha$ имеет место $(\mathfrak{B} \rightarrow \mathfrak{2})_{-\alpha}$, a для некоторого $\beta \geqslant \alpha-(\mathfrak{B})_{\beta}$ и $(\mathfrak{U})_{-\beta}$, а также $\left(\mathfrak{B}_{v}\right)_{\beta}$ для каждого подмножества $v$ множества элементарных типов нашей сигнатуры.

Построим теперь модель $M^{\prime}$ следующим образом. В качестве семантической структуры выберем аспект $\beta$ вместе с его конкретизациями и направлениями, им принадлежащими. Для построения системы объектов сначала возьмем те объекты, которые являются значениями констант формулы $\mathscr{A}$ в $(\mathfrak{U})_{-\beta}$, а затем добавим новые объекты с помощью следующих операций:

1) если в $M$ для $\gamma \geqslant \beta$ и некоторой умеренной формулы вида $\forall\left\langle x_{\imath}^{\prime}: \imath \in I\right\rangle \Subset$ имеет место $\left.\left(\forall\left\langle x_{1}^{\prime}: \imath \in I\right\rangle\right) \mathcal{E}\right)_{-\gamma}$ и значения констант в $ᄃ$ содержатся в $M^{\prime}$, то дополним ее объектами $\left\langle a_{\imath}: \iota \in I\right\rangle$ такими, что $\left(厄\left(a_{\imath}: \iota \in I\right)\right)_{-\delta}$ при некотором $\delta \geqslant \gamma$;

2) если в $M$ для $\gamma \geqslant \beta$ и некоторой умеренной формулы вида $\mathbf{\Xi}\left\langle x_{\imath}^{\prime}: \iota \in I\right\rangle \mathcal{E}$ имеет место $\left(\boldsymbol{\Xi}\left\langle x_{1}^{\prime}: \iota \in I\right\rangle \in\right)_{\gamma}$ и значения констант в $ᄃ$ содержатся в $M^{\prime}$, то дополним каждый аспект $\delta$ из некоторого множества, исчерпывающего аспект $\gamma$, объектами $\left\langle a_{\imath}: \iota \in I\right\rangle$ такими, что $\left(\mathcal{C}\left(a_{2}: \iota \in I\right)\right)_{8}$;

3) если для умеренной формулы (5 со свободными переменными $\left\langle x^{\prime}{ }_{\imath}: \imath \in I\right\rangle$ значения констант формулы $ᄃ$ содержатся в аспекте $\gamma \geqslant \beta$ модели $M^{\prime}$, то дополним ее объектом $P$ такого же типа, для которого в $M$ имеет место $\left(\mathrm{V}\left\langle x_{2}^{\prime}: \iota \in I\right\rangle\left(\Subset \leftrightarrow P\left\langle x_{\tau}^{\prime}: \iota \in I\right\rangle\right)\right)_{\gamma}$;

4) если $P_{1}$ и $P_{2}$ принадлежат одному неэлементарному типу и оба имеются в аспекте $\gamma$ модели $M^{\prime}$, то дополним ее семейством объектов $\left\langle a_{\imath}: \iota \in I\right\rangle$ такого же типа, которое содержит не более чем $\aleph$ (конечное число) разных объектов элементарных типов и для которого существует $\delta \geqslant \gamma$ такой, что $\left(P_{1}\left\langle a_{2}: \iota \in I\right\rangle \leftrightarrow P_{2}\left\langle a_{2}: \iota \in I\right\rangle\right)_{-\delta}$ и $\left(\left[a_{2}\right]^{*}\right)_{\delta}$ для всех объектов $a_{2}$ неэлементарного типа (если такое семейство $\left\langle a_{i}: \iota \in I\right\rangle$ существует).

Множество применений всех этих операций вполне упорядочено, причем в случае операции 2) вполне упорядочено и множество аспектов $\delta$. Индивиды модели $M^{\prime}$ существуют в тех же аспектах, что и в модели $M$, а объекты $а$ неэлементарного типа можно в $M^{\prime}$-считать существующими только в тех аспектах, где в $M$ имеет место $[a]^{*}$.

В ходе построения модели $M$ новые индивиды добавляются на каждом шаге только в том случае, если индивидов, добавленных на предыдущих шагах (в смысле данного полного упорядочения), недостаточно для выполнения условия операции. В случае операций 1) и 4) это значит, что для $\varepsilon \geqslant \delta$ не существует семейства, содержащего только добавленные ранее индивиды и выполняющего в $\varepsilon$ условие, требуемое в $\delta$ для семейства $\left\langle a_{2}: \iota \in I\right\rangle$. В случае операции 2) это значит, что никакое $\delta$ из указанного множества не исчерпывается множеством $\varepsilon$ таких аспектов, где существуют семейства, содержащие 
только добавленные ранее индивиды и выполняющие в $\varepsilon$ условие, требуемое в $\delta$ для семейства $\left\langle a_{\imath}: \iota \in I\right\rangle$.

Возникает умеренно нормальная модель, где все умеренные формулы имеют то же истинностное значение, что и в модели $M$. Поэтому эта модель является контрмоделью для $\mathfrak{*}$ и, по теореме 2, ее индивиды должны составлять собственный класс. Каждый из этих индивидов входит в состав некоторого семейства $\left\langle a_{\imath}: \iota \in I\right\rangle$ объектов, добавленного на некотором шаге для некоторого аспекта $\delta$. А так как аспекты составляют множество, то для некоторого аспекта $\delta$ добавлен собственный класс таких семейств.

Для семейств, добавленных к этому $\delta$ операциями 1), 2) и 4), определим понятие сохранения характера семейства. Для операций 1) и 4) характер семейства считается сохраненным в тех $\varepsilon \geqslant \delta$, где выполнено условие, поставленное для семейства в $\delta$, т. е. где имеет место

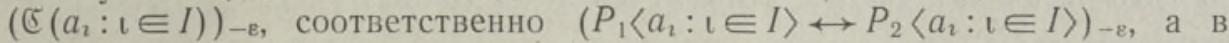
случае операции 2) - в тех $\varepsilon \geqslant \delta$, где $\varepsilon$ не исчерпывается множеством аспектов, содержащих некоторое семейство $\left\langle b_{\imath}: \iota \in I\right\rangle$, состоящее только из таких индивидов, которые введены раньше семейства $\left\langle a_{i}: \iota \in I\right\rangle$, и удовлетворяющее $\Subset\left(b_{\imath}: \iota \in I\right)$.

Для каждого из этих семейств $\left\langle a_{\imath}: \iota \in I\right\rangle$ обозначим через $E\left\langle a_{\imath}: \iota \in I\right\rangle$ множество тех аспектов $\varepsilon \geqslant \delta$, где сохраняется характер семейства $\left\langle a_{\imath}: \iota \in I\right\rangle$. Так как разные множества $E\left\langle a_{\imath}: \iota \in I\right\rangle$ составляют множество, а семейства $\left\langle a_{\imath}: \iota \in I\right\rangle-$ собственный класс, то имеется некоторый собственный класс данных семейств $\left\langle a_{2}: \iota \in I\right\rangle$ таких, что для каждого из них $E\left\langle a_{\imath}: \iota \in I\right\rangle$ одно и то же.

Из этого класса выбираем последовательность длины $\aleph^{+}$(счетную последовательность), содержащую семейства $\left\langle a_{2}: \iota \in I_{\mu}\right\rangle, \mu \in \pi, \quad$ в порядке использования их операциями добавления объектов. Объединяем индивиды этих семейств в одно семейство без повторений, т. е. опуская повторяющиеся индивиды. Так как в каждое семейство попадает хотя бы один новый индивид, то получим семейство $\left\langle a_{\tau}: \tau \in J\right\rangle$ длины $\boldsymbol{\aleph}^{+}$(счетное). Так как $\delta \geqslant \beta$, то имеет место $\left(\mathfrak{B}_{\nu}\right)_{\delta}$ для каждого подмножества $v$ множества элементарных типов. Выбираем в качестве $v$ множество типов индивидов $a_{\tau}, \tau \in J$. Варьируем квантор общности в начале $\mathfrak{B}_{v}$ так, чтобы были использованы все индивиды семейства $\left\langle a_{\tau}: \tau \in J\right\rangle$. Затем реализуем квантор существования и дизъюнкцию в начале $\mathfrak{B}_{v}$, но так, чтобы некоторое, а значит, и каждое семейство $\left\langle a_{\imath}: \iota \in I_{\mu}\right\rangle, \mu \in \pi$, сохраняло свой характер. При этом мы попадем в некоторый $\varepsilon \in E\left\langle a_{\imath}: \iota \in I_{\mu}\right\rangle$, где имеются индивиды $b_{\tau}, \tau \in J$, среди которых не более чем $\aleph$ (конечное число) разных, так что $\left(\nabla P\left(P\left\langle a_{x}: x \in K\right\rangle \leftrightarrow P\left\langle b_{\varkappa}: x \in K\right\rangle\right)\right)_{\varepsilon}$ для каждого $K \subseteq J$ мощности

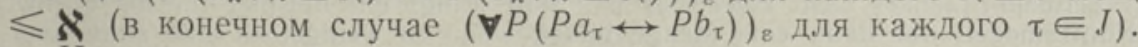

Но из-за соотношения мощностей множества $J$ и множества разных индивидов $b_{\tau}, \tau \in J$, существует $\tau_{0} \in J$ такой, что все индивиды $b_{\tau}$ уже представлены в семействе $\left\langle b_{\tau}: \tau<\tau_{0}\right\rangle$. Следовательно, если для каждого $\mu \in \pi$ семейство $\left\langle b_{\imath}: \iota \in I_{\mu}\right\rangle$ получено из семейства $\left\langle a_{\imath}: \iota \in I_{\mu}\right\rangle$ заменой индивидов $a_{\imath}=a_{\tau}, \iota \in I_{\mu} ; \tau \in J$, на соответствующие индивиды $b_{\tau}, \quad \tau \in J$, то существует $\mu_{0} \in \pi$ такое, что индивиды семейства $\left\langle b_{2}: \iota \in I_{\mu_{0}}\right\rangle$ уже фигурируют в объединении семейств $\left\langle b_{\imath}: \iota \in I_{\mu}\right\rangle$. $\mu<\mu_{0}$. Пусть $K \sqsubseteq J-$ множество таких $\tau$, что каждый индивид $b_{\imath}: \iota \in I_{\mu_{0}}$ содержится в семействе $\left\langle b_{\tau}: \tau \in J\right\rangle$ впервые на месте $\tau_{2} \in K$.

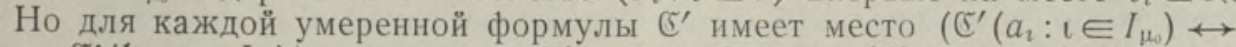
$\left.\leftrightarrow \mathcal{S}^{\prime}\left(b_{\imath}: \iota \in I_{\mu_{0}}\right)\right)_{\varepsilon}$, а также $\left(\mathcal{C}^{\prime}\left(a_{\tau_{\imath}}: \iota \in I_{\mu_{0}}\right) \leftrightarrow \mathcal{S}^{\prime}\left(b_{\tau_{\imath}}: \iota \in I_{\mu_{0}}\right)\right)_{\varepsilon}$ (в конечном случае $I_{\mu_{0}}$ конечно и так как для формул с одной свободной переменной эквивалентность имеет место, то через конечное число шагов получим, что она имеет место и для любой данной формулы с конечным числом свободных переменных). Но так как $b_{\imath}=b_{\tau_{\text {i }}}$, то 
$\left(\mathcal{E}^{\prime}\left(a_{\imath}: \iota \in I_{\mu_{0}}\right) \leftrightarrow \mathbb{C}^{\prime}\left(a_{\tau_{\imath}}: \iota \in I_{\mu_{0}}\right)\right)_{\varepsilon}$. Но это невозможно, если в качестве $\widetilde{\digamma}^{\prime}$ взять $匚$ в случае введения семейетва $\left\langle a_{\imath} \iota \in I_{\mu_{0}}\right\rangle$ операцией 1) или $2)$, или $\left(P_{1}\left\langle x_{\imath}: \iota \in I_{\mu_{0}}\right\rangle \leftrightarrow P_{2}\left\langle x_{\imath}: \iota \in I_{\mu_{0}}\right\rangle\right)$ в случае операции 4).

Полученное противоречие доказывает теорему.

Таким образом, мы доказали, что если не для каждой формулы имеет место случай 1) из случаев, указанных в начале статьи, то существуют и такие формулы, для которых обязательно имеет место случай 3).

\title{
Л ИТЕР А Т У Р А
}

1. Tаутс А. Уч. зап. Тартуск. ун-та, № 556, 3-10 (1981).

2. Tаутс A. Уч. зап. Тартуск. ун-та, № 390, 29-42 (1976).

3. Tаутс A. Уч. зап. Тартуск. ун-та, № 390, 3-28 (1976).

4. Tаутс А. Уч. зап. Тартуск. ун-та, № $355,7-19$ (1975).

\author{
Ннститут кибернетики \\ Академии наук Эстонской ССР \\ Поступила в редакцию \\ 8 июля 1982
}

\section{A. TAUTS}

\section{VOIMALUSEST EHITADA KONTRAMUDEL MITTEDETERMINEERITUD VALEMILE}

Artiklis on näidatud, et kui leidub selliseid mittedetermineeritud valemeid $\left[{ }^{3}\right]$, millel ei ole kontramudelit hulga näol, siis on ka selliseid mittedetermineeritud valemeid, mille igas kontramudelis on pärisklass aspekte.

\section{A. TAUTS \\ OBER DIE MÖGLICHKEIT, ZU EINER INDETERMINIERTEN FORMEL EIN KONTRAMODELL ZU KONSTRUIEREN}

Im vorliegenden Artikel werden Kontramodelle für indeterminierte Formeln $\left[{ }^{3}\right]$ auf der Basis der semantischen Struktur $\left.{ }^{[}{ }^{1}\right]$ betrachtet. A priori kann man drei Fälle unterscheiden: 1) zu jeder indeterminierten Formel kann man ein solches Kontramodell konstruieren, in dem die Aspekte und die Individuen eine Menge bilden; 2) zu jeder indeterminierten Formel kann man ein solches Kontramodell konstruieren, in dem die Aspekte eine Menge bilden, aber es gibt eine Formel, für die in diesem Fall die Individuen eine echte Klasse bilden; 3) es gibt eine Formel, für die in jedem Kontramodell die Aspekte eine echte Klasse bilden. Es stellt sich heraus, daB der Fall 1) auf Grund der Aksiomatik von Zermelo-Fraenkel nicht beweisbar ist. Man bringt ein Beispiel für die Formel, deren Kontramodell nur dann eine Menge bildet, wenn es wenigstens eine stark unerreichbare unzählbare Kardinalzahl gibt. Man ḅeweist, daß falls es bei einer Formel nicht möglich ist, dazu ein Kontramodell zu konstruieren, das eine Menge wäre, d. h. wenn der Fall 1) nicht stattfindet, dann gibt es auch eine solche indeterminierte Formel, bei der die Aspekte des Kontramodells bestimmt eine echte Klasse bilden, d.h. es gilt der Fall 3). 